EPJ manuscript No.

(will be inserted by the editor)

\title{
Extended event driven molecular dynamics for simulating dense granular matter
}

\author{
J. Sebastián González ${ }^{1,2}$, Dino Risso ${ }^{3}$, and Rodrigo Soto ${ }^{1}$ \\ 1 Departamento de Física, FCFM, Universidad de Chile, Santiago, Chile. \\ 2 Faculty of Engineering Technology, TS, MSM, University of Twente, Enschede, Netherlands. \\ 3 Departamento de Física, Universidad del Bío-Bío, Concepción, Chile.
}

\begin{abstract}
A new numerical method is presented to efficiently simulate the inelastic hard sphere (IHS) model for granular media, when fluid and frozen regions coexist in the presence of gravity. The IHS model is extended by allowing particles to change their dynamics into either a frozen state or back to the normal collisional state, while computing the dynamics only for the particles in the normal state. Careful criteria, local in time and space, are designed such that particles become frozen only at mechanically stable positions. The homogeneous deposition over a static surface and the dynamics of a rotating drum are studied as test cases. The simulations agree with previous experimental results. The model is much more efficient than the usual event driven method and allows to overcome some of the difficulties of the standard IHS model, such as the existence of a static limit.
\end{abstract}

\section{Introduction}

Historically, hard sphere models have been very important in understanding some physical processes. This is because they allow for a direct comparison between the different theoretical approaches and precise numerical simulations [1]. For example, hard sphere models of atoms helped in understanding the development of short range correlations in liquids and the different roles played by the repulsive and attractive components of the interatomic potential energy [2. Similarly, poly-disperse hard sphere particles helped to improve understanding of the jamming transition in glasses 3 .

In a simplified, but extremely useful description of granular matter, grains are considered as hard spheres. The energy dissipation through collisions is included via a restitution coefficient, constituting the so-called inelastic hard sphere (IHS). In its simplest form, the restitution coefficient is constant and affects only the normal relative velocities, hence modeling soft spheres. More realistic collisions, including tangential restitution coefficients, static and dynamic friction, and rotation have been considered 4. The IHS model, in its different forms, has been very successful on describing the main features of granular flows and it has become a useful tool to improve understanding of the different phenomena that appear in granular matter (see, for example [5, 6, 7]).

Hard sphere models, in general, are numerically simulated by using the event driven molecular dynamics (EDMD) method. This method consists in repeating the following procedure: first to predict the next collision event between two particles or with the walls, then to analytically bring forward the system to that instant, and finally compute the velocities of the particles after the collision. That is, the system advances from event to event. This method is very precise because there is no time discretization of the integration of trajectories or collisions, as the particle dynamics can be solved analytically between collisions. It is also efficient because the effective time step between events per particle matches the mean free time. Furthermore, after 
several optimizations it takes a time $\mathcal{O}(\log N)$ to attend every event, where $N$ is the number of particles 8 .

It was soon realized that the simulation of IHS particles presented the inelastic collapse problem [9: inelasticity increases the probability of recollisions and it is possible that few of the grains perform an infinite number of collisions among themselves in a finite physical time. Obviously, infinite events must be attended taking an infinite amount of computational time. This phenomenon is an artifact of the IHS model in which collisions are instantaneous while in Nature collisions take a small, but finite time. This artifact is corrected by regularizing the model: inelastic collisions are turned into elastic ones in the case where the relative velocity is small [9] or when the collision frequency goes above a certain threshold [10. However, when simulating systems where dense regions coexist with fluid-like regions under the presence of gravity the regularized IHS models becomes very inefficient. In dense regions, due to the regularization, particles remain colliding elastically with small relative velocities and high frequencies, forming solid-like structures. All these collisions produce almost no dynamic evolution, but nevertheless constitute the overwhelming majority of all collisional events in the system.

Commonly, to overcome this difficulty, the simulation of dense flows is performed using time driven molecular dynamics of continuous force models like the Hertzian contacts, the springdashpot model, or the ratchet model [11. However, realistic values of the Young modulus need the use of extremely small time steps, making the simulations extremely costly. Sometimes, to speed up simulations, smaller values of the Young modulus are used. Other approach is the method of contact dynamics, in which the static reaction forces between grains are obtained as a solution of a large set of equations at a considerable computational cost [12.

In this article we present an extension to the IHS model of granular systems, that remains efficient in situations where solid-like and fluid regions coexist in presence of gravity and confining walls. It has been reported [10 that the IHS model has three major drawbacks: 1) The number of collisions can diverge, i.e. the inelastic collapse can occur; 2) all interactions are binary, multiparticle contacts cannot occur; and 3) no static limit exists. Besides there are no persistent contacts, as happens in dense flows. Our model proposes a solution to these problems except for the second one, which is only considered partially because binary collisions is indeed one of the key ingredients that allows to perform efficient simulations.

\section{Simulation strategy}

Consider the simple case of a particle bouncing inelastically against a static wall. If $\alpha$ is the restitution coefficient with the wall, $V_{0}$ is the velocity just before the first bounce, and $g$ is the gravity, the particle would stop at a time $t^{*}=2 V_{0} /(1-\alpha) g$ after an infinite number of collisions [13. If, to avoid this inelastic collapse, the model is regularized such that collisions were set to elastic when velocities become smaller that $V_{c}$, the particle would remain colliding very frequently for an infinite time, with an irrelevant dynamics. The solution we propose is that, when the velocity reaches $V_{\text {sleep }}>V_{c}$, the particle is asleep and becomes frozen. In this state the particle does not move and does not accelerate either; physically, this is possible because the particle is supported on the wall. To avoid sleeping a particle at exactly contact with another, which would lead to ill defined dynamics when awake, it is asleep in an advanced position (halfway to the next collision that it would have had if it continues with the normal dynamics). Figure 1 summarizes the described collision sequences.

Therefore, for granular flows, we generalize the state of the particles to indicate the type of dynamics they follow: normal or frozen. Normal particles have the regularized IHS dynamics, while frozen particles do not move or accelerate either with respect to a fixed ground. For the sake of simplicity we will call sleep to the pass of a particle from normal to frozen state, and wake up to the contrary. To simulate this extension to the IHS model we will use the EDMD method where sleeping and waking up particles will be treated as events as well. The key point is to find efficient, as well as physically sensible, criteria to sleep or wake up particles. These criteria should be local in time and space to maintain the efficiency of the event driven algorithm.

A particle is asleep if its velocity is smaller than a threshold value $V_{\text {sleep }}$ and it is in a mechanically stable position. To check this last condition, without making a detailed force 


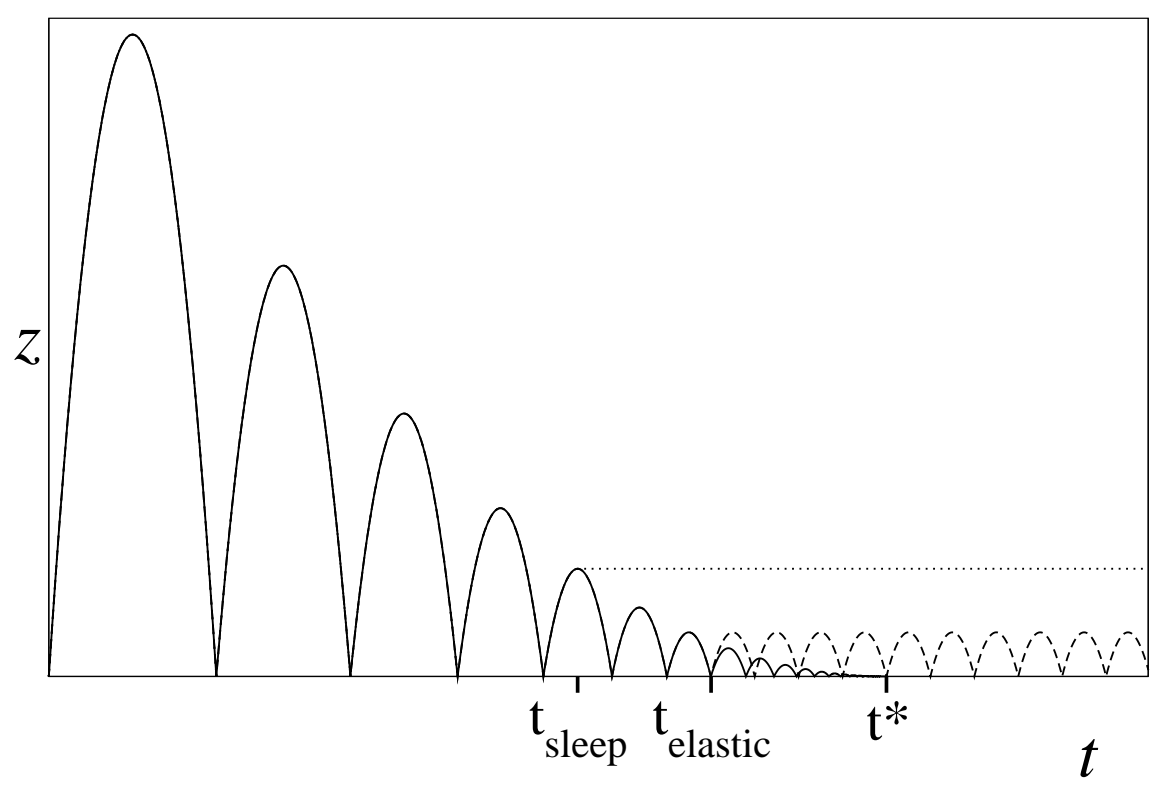

Fig. 1. Height of a particle bouncing inelastically against a static wall. With the full inelastic dynamics the particle stops after infinite collisions at $t^{*}$ (solid line). If, at $t_{\text {elastic }}$, collisions are turn into elastic for small velocities, the particle continues bouncing indefinitely (dashed line). Our model sleeps the particle if the velocity is small enough at $t_{\text {sleep }}$ and after that it remains motionless (dotted line). The sleeping time $t_{\text {sleep }}$ is located in a slightly later time than the instant in which $V<V_{\text {sleep }}$ such that the particle is not exactly at contact with the wall.

analysis, which is anyway ill defined in hard sphere models, we propose a heuristic method. A particle approaching a mechanically stable position proceeds with a series of collisions with other particles where, at least one of them, is already a frozen particle. When approaching this equilibrium position the velocity must be decreasing but, to avoid to erroneously identify a particle that is climbing over another, it is also demanded that the particle is going down. In summary a particle is asleep if when colliding with a frozen particle: (a) it is going down and (b) its velocity is smaller than at the previous collision and smaller than $V_{\text {sleep. }}$. Sleeping particles partially solves one of drawbacks of the IHS model: frozen particles have multiple contacts but normal ones only have binary interactions.

Note that when sleeping a particle, it is not necessary that all particles below it are already frozen but just one. With this approach it is possible to build up arches as represented in Fig. 2. a fundamental object in granular solids. This possibility is ruled out in other algorithms like the bottom-to-top [14].

In Fig. 2 particle 2 is asleep in (b) fulfilling all conditions, which is only possible if particle 3 is converging to equilibrium as well. But using of a finite value for $V_{\text {sleep }}$ could consider particle 3 to be converging while it is in a marginally unstable position. That is, a particle could be erroneously asleep on top of another in a marginally unstable position although its velocity would have seemed to converge to zero. However, this problem is solved by the self-check protocol described later.

Intuitively, to wake up a frozen particle, it must be hit by another particle with high enough velocity. However, as the frozen particle is supported over others, the collision process is complex and some heuristic must be taken. For simplicity a frozen particle is awake if it is hit with a velocity larger than $V_{\text {wakeup }}$; otherwise the collision is performed as if the mass of the frozen particle is infinite and there is a restitution coefficient $\alpha_{\text {frozen }}<\alpha$, which models in a simplified way the multiple collisions inside the frozen region 15. Below, it is shown that, besides the collisional method to wake up a particle, they must be self-checked and waken up spontaneously if they loose their mechanical stability. 
(a)

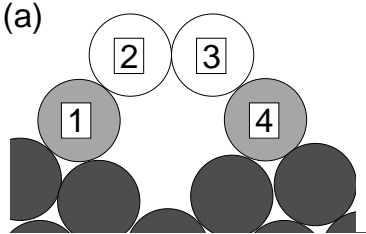

(b)

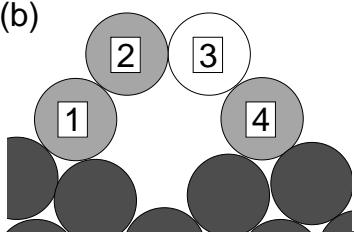

(c)

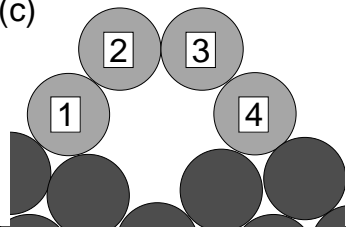

Fig. 2. Dynamic sequence in two dimensions allowing to build up arches of frozen particles. In (a) normal particles 2 and 3 have velocities converging to zero through a series of frequent collisions between themselves and with particles 1 and 4, which are frozen and already supported over others; in (b) 2 sleeps after a collision with 1; finally in (c) 3 sleeps after a collision with 2 or 4 . The arch can also be built by sleeping first 3 and then 2 .

Sleeping a particle is an inelastic process because its energy, although small, is lost. If there is a sequence of repeated sleeping and waking up processes, an inelastic collapse can occur. This sequence can take place when a particle that is on top of a frozen region is collided frequently from above as in avalanches or in a deposition over a surface. To avoid this inelastic collapse, the energy that the particle had when it was asleep is stored for a time of the order of $2 V_{\text {sleep }} /\left(1-\alpha_{\text {frozen }}\right) g$, that is, the inelastic collapse time over a frozen region. If it is awake before that time this energy is reinjected.

A note should be done regarding the prediction of the collision time between grains, that is obtained from the equation $\left|\mathbf{r}_{1}(t)-\mathbf{r}_{2}(t)\right|=d$. The relative distance between two normal grains does not depend on gravity and the equation is quadratic, which is simply solved. But in the case of a normal and a frozen particle the equation becomes quartic and good root finding methods should be used to avoid missing solutions, i.e. collisions. In the case of a normal particle that just collided with a frozen one, the prediction of the recollision time reduces to a cubic equation because one solution is already known (the present time).

The particles diameter $d$ and the gravitational acceleration $g$ define natural microscopic units for time and velocity, $\sqrt{d / g}$ and $\sqrt{g d}$, respectively. The threshold velocities must be small compared with this velocity scale. To avoid the inelastic collapse in the bulk we set $V_{c}=10^{-4} \sqrt{g d}$.

In order to test the model in demanding regimes, we have considered quite inelastic systems where the interparticle restitution coefficient is fixed to $\alpha=0.7$. In absence of a more refined model for the particle-solid restitution coefficient its value is fixed arbitrarily to a small value $\alpha_{\text {frozen }}=0.4$.

\section{Homogeneous deposition}

First, we consider the homogeneous deposition of grains in a container to obtain a granular packing. Different methods have been used in the literature for that purpose: (a) In the the bottom-to-top reconstruction method, particles are added one-by-one waiting to the previous one to have found an equilibrium position [14. This method is unable to form arches and therefore it cannot reproduce all the phenomenology of granular solids. Besides, the kinetic energy of the particles is lost and it is not possible for a particle to wake up others. (b) The simulation of grains with dissipative Hertz contacts needs small time steps to resolve collisions. Hence, it becomes extremely time consuming when realistic values of the Young modulus are used. (c) In Ref. [16] grains grow until a jamming situation is attained, representing a different physical mechanism.

Here, we let $N=4800$ particles to fall down in an homogeneous rain at a small volume fraction $(\phi \simeq 0.04)$ in a box of size $10 d \times 10 d \times 500 d$. Each particle falls at a constant vertical velocity $V_{\text {fall }} \simeq-2.5 \sqrt{g d}$, until it has its first collision. From then on the particle adopts the dynamics described in the previous section. This is analog to a deposition with a moving heap keeping constant the distance between the heap and the free surface [17. The base is composed of a random packing of frozen grains that never wake up, in order to avoid a local crystalline order 
in the lower layers. Periodic boundary conditions are used in the lateral directions. Simulations stop when all particles are frozen with zero kinetic energy.

In Fig. 3 we present the evolution of the number of normal and frozen grains in a simulation made with $V_{\text {sleep }}=V_{\text {wakeup }}=0.01 \sqrt{g d}$. The number of normal particles, which are the effectively simulated ones, only occupy a small region on top of the deposit and it remains roughly constant as the deposited layer grows (see Fig. 4). The average number of normal particles is $\left\langle N_{\text {normal }}\right\rangle=280 \pm 2$. Considering a packing fraction equal to that of the random close packing, $\mathrm{RCP}, \phi_{\mathrm{RCP}}=0.64$, this corresponds to a fluidized layer of $4.4 d$ in height.

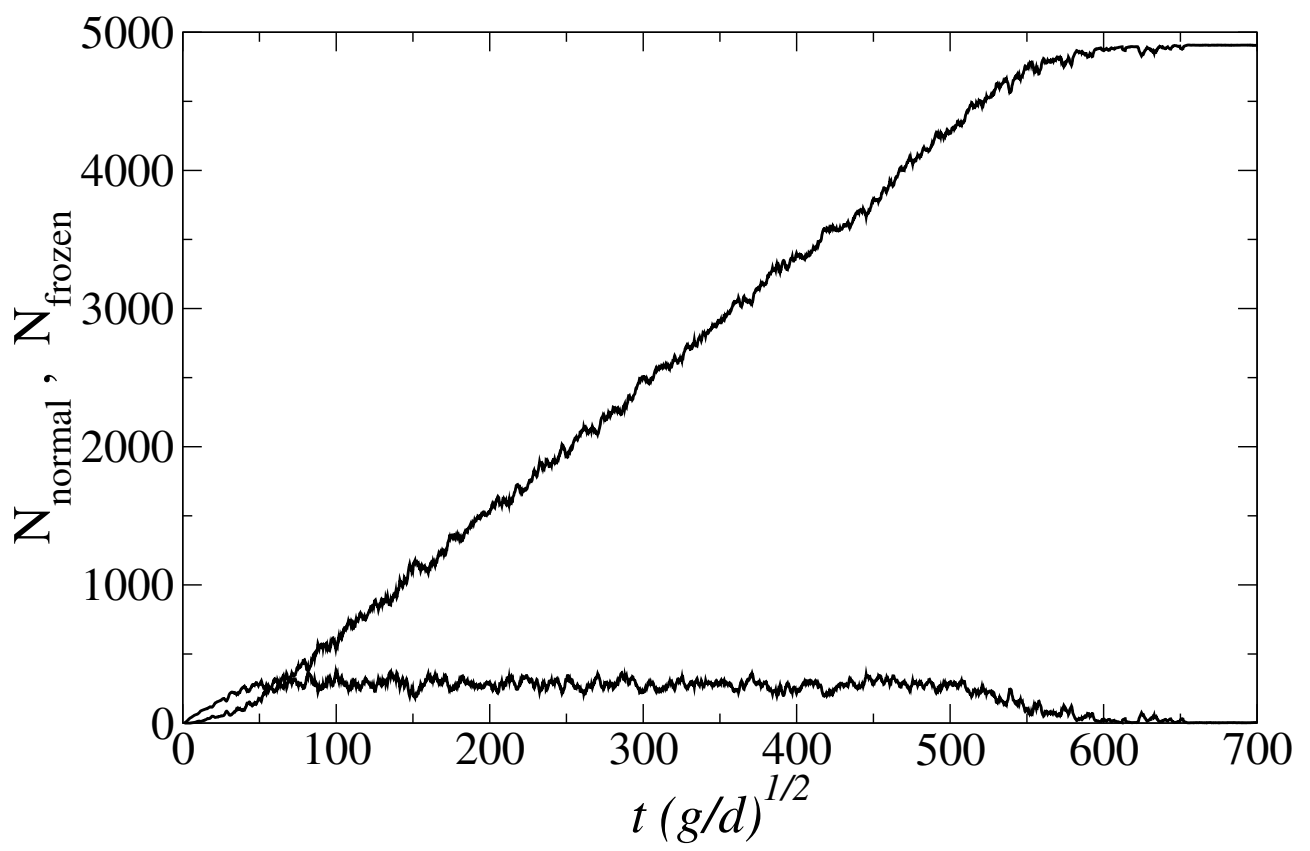

Fig. 3. Evolution of the number of normal (lower curve) and frozen (upper curve) particles during an homogeneous deposition. Particles with constant speed are not included in the graph.

Figure 5 presents the CPU time needed to achieve different simulation times for three values of the threshold velocities with $V_{\text {sleep }}=V_{\text {wakeup. }}$ It is seen that when then threshold velocities are finite, the CPU time grows linearly with the simulation time as the normal particles remain constant through all the simulation as seen in Fig. 3 although the proportionality constant depends on the value of the threshold velocities as more particles are normal when they have smaller thresholds. In the case of vanishing thresholds the CPU time grows at least quadratically with the simulation time as the number of normal particles continuously increases.

A series of simulations were run varying the threshold velocities. For simplicity, we choose $V_{\text {sleep }}=V_{\text {wakeup }}=V_{t}$ in the range $0.01 \leq V_{t} / \sqrt{g d} \leq 0.1$. The packing fraction, computed in the middle region of the deposit, increases slightly when the threshold velocities become smaller and it is well fitted by the expression $\phi=(0.640 \pm 0.001)-(0.11 \pm 0.01) V_{t} / \sqrt{g d}$. The asymptotic packing fraction with the full dynamics is $\phi_{0}=0.640$, equal to the random close packing value $\phi_{\mathrm{RCP}}=0.64$ 16 and largely above the random loose packing value $\phi_{\mathrm{RLP}}=0.55518$. The RCP limit could be attained due to the upper layer that remains in a fluid state, allowing to efficiently accommodate the grains thanks to the energy transfer from the deposited grains to the ones that were frozen. On the contrary, if the fluidization layer is avoided, for example, by depositing particles one-by-one and setting $V_{\text {wakeup }}=\infty$ and $V_{\text {sleep }}=0.05 \sqrt{g d}$ (like in the bottom-to-top algorithm [14]), the packing fraction is much lower: $\phi_{\text {onebyone }}=0.586$.

When simulating packings, a balance should be assumed when choosing the threshold velocities in order to obtain accurate values for the packing fraction at an affordable computational 

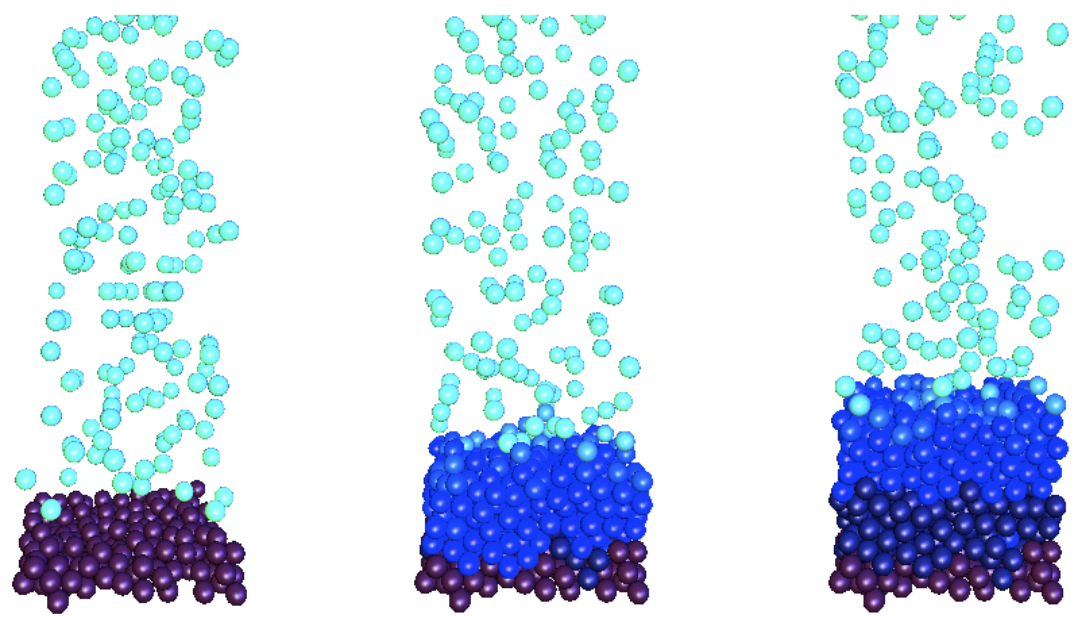

Fig. 4. Three snapshots of the deposition process for $V_{\text {sleep }}=V_{\text {wakeup }}=0.01 \sqrt{g d}$ at different instants: left $t=0 \sqrt{d / g}$, center $t=50 \sqrt{d / g}$, and right $t=100 \sqrt{d / g}$. The base particles that cannot wakeup are presented in purple, particles in dark blue are frozen, the particles of the homogenous rain are presented in light blue, and the normal particles are presented in different levels of blue depending on their kinetic energy.

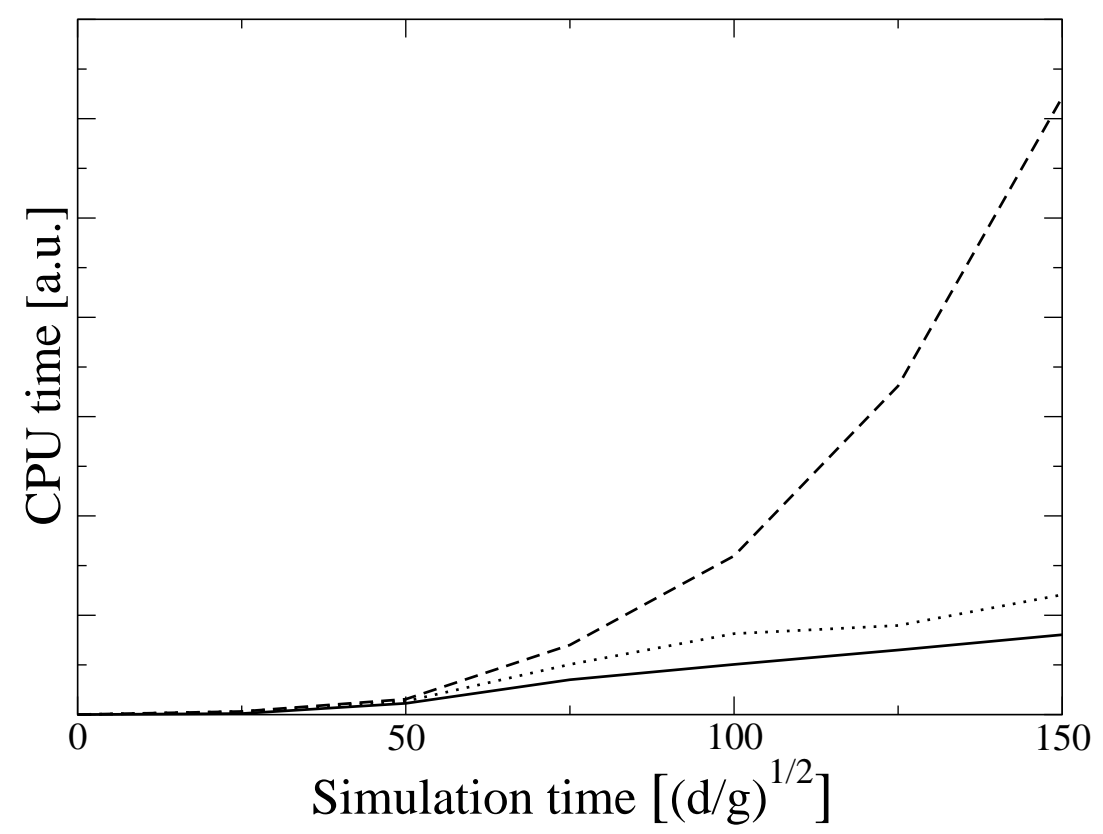

Fig. 5. CPU time, in arbitrary units, to achieve different simulation times for three values of the thresholds $V_{t}=V_{\text {sleep }}=V_{\text {wakeup }} . V_{t}=0.0$ (dashed line), $V_{t}=0.05 \sqrt{g d}$ (dotted line), and $V_{t}=0.10 \sqrt{g d}$ (solid line).

cost. In our runs the total number of events performed until the simulation stops diverges as $N_{\text {events }} \sim V_{t}^{-1.2}$. It should be noted that the packing fraction dependence is much softer than the computational cost, and therefore in normal applications high enough threshold values can be taken. 


\section{Rotating drum and the self-check protocol}

In the case of the simple deposition described above, it is not crucial the chosen value of $V_{\text {wakeup }}$ because the final state is motionless. A well studied regime where particles change continuously from normal to frozen, and where we can study the effect of the wake-up threshold in the dynamics, is the rotating drum 19.

We consider a half-filled cylinder of radius $R=25 d$, thickness $L=5 d$, with periodic boundary conditions in this direction, and fixed angular frequency $\Omega=0.075 \sqrt{g / d}$. The rotation of the drum is modeled by changing the direction of the gravitational force at a fixed rate. Doing this, we can emulate moving walls without actually have to move them. In the case of moving the walls, the velocity thresholds would refer to the relative velocity of the colliding particles and not to the absolute velocities. This approach neglects the centrifugal forces, that are small for the angular velocity we use. The drum wall is made of a regular array of frozen particles that cannot wake up, with a transverse and arc separation of $1.05 d$ and $1.0 d$, respectively (see Fig. 6).
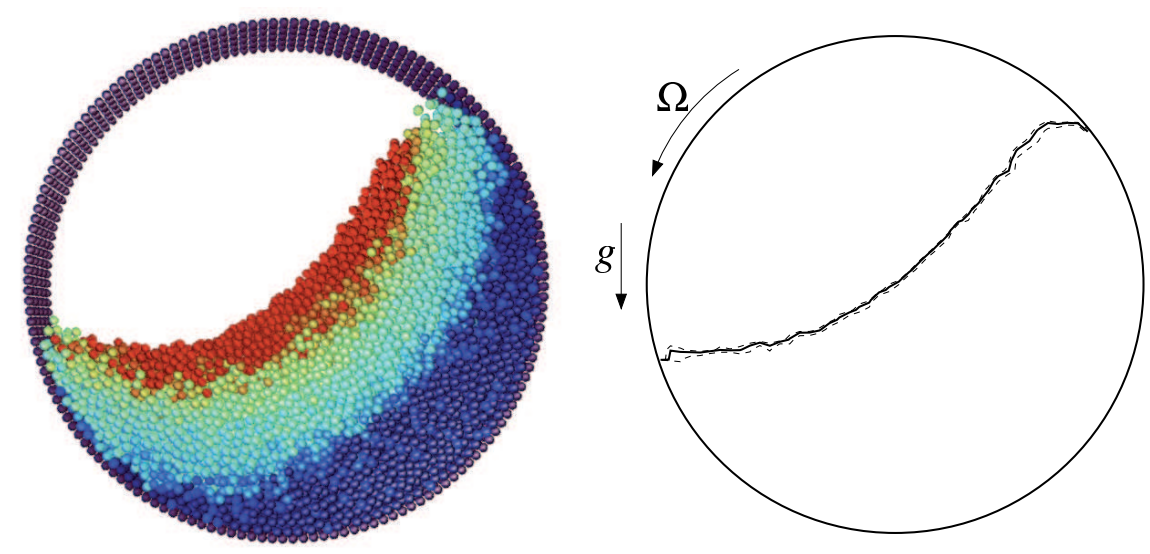

Fig. 6. Left: Snapshot of the rotating drum in the stationary state. Colors indicate the kinetic energy. Right: Average packing fraction iso-lines. The solid line is for $\phi=0.5 \phi_{R C P}$ and the dashed lines are for $\phi=0.25 \phi_{R C P}$ and $\phi=0.75 \phi_{R C P}$.

Avalanches form in the rotating drum because particles that are raised to the top loose their mechanical stability. However, the collisional wake up mechanism described in section 2 is not able to wake them up under this condition. To overcome this problem, a self-check protocol is set: each particle is checked every $\tau_{\text {check }}$ to verify that all the particles that were supporting it when it became frozen are still there. According to Fig. 2, a particle is supported over neighbor particles (closer than 1.05d) that are frozen below it (defined in terms of the direction of gravity) and eventually of normal type above it. Note that, a change in the direction of gravity could turn a mechanically stable configuration into an unstable one. If the test fails the particle is waken up; if it is still in a mechanically stable position it will sleep again after a few collisions, otherwise it will continue in the normal state. The self-check mechanism allows also to wake-up particles that were erroneously asleep on top of others in marginally unstable positions.

In the simulation of the deposition, $\tau_{\text {check }}$ was set to infinity because in that setup, no particle will loose its mechanical stability once settled down. In the present case, a small value of $\tau_{\text {check }}$ would lead to too many particles being checked, increasing the computational cost and eventually to an inelastic collapse; a large value of it would artificially maintain particles in mechanically unstable positions. As a compromise we have chosen $\tau_{\text {check }}=0.1 \sqrt{d / g}$.

The particles that are waken up after the self-check and continue to be normal will avalanche through the inclined free surface, waking up other particles through collisions. Figure 6 presents a typical snapshot of the system once the system has reached the stationary state. Some packing fraction iso-lines, measured by averaging different snapshots of two compete rotations of the 
drum, are presented in the same figure. A clear free surface develops with an abrupt density drop.

In order to show the effect of $V_{\text {wakeup }}$ in the dynamics, we present in Fig. 7 the velocity profiles in the reference frame of the rotating drum, for fixed $V_{\text {sleep }}=0.05 \sqrt{g d}$ and different values of $V_{\text {wakeup. }}$ These profiles should be compared with the experimental results reported in 19, which show a linear shear band followed by a frozen region that is static with respect to the drum. In between, there is a small transition region, where velocity decays exponentially.

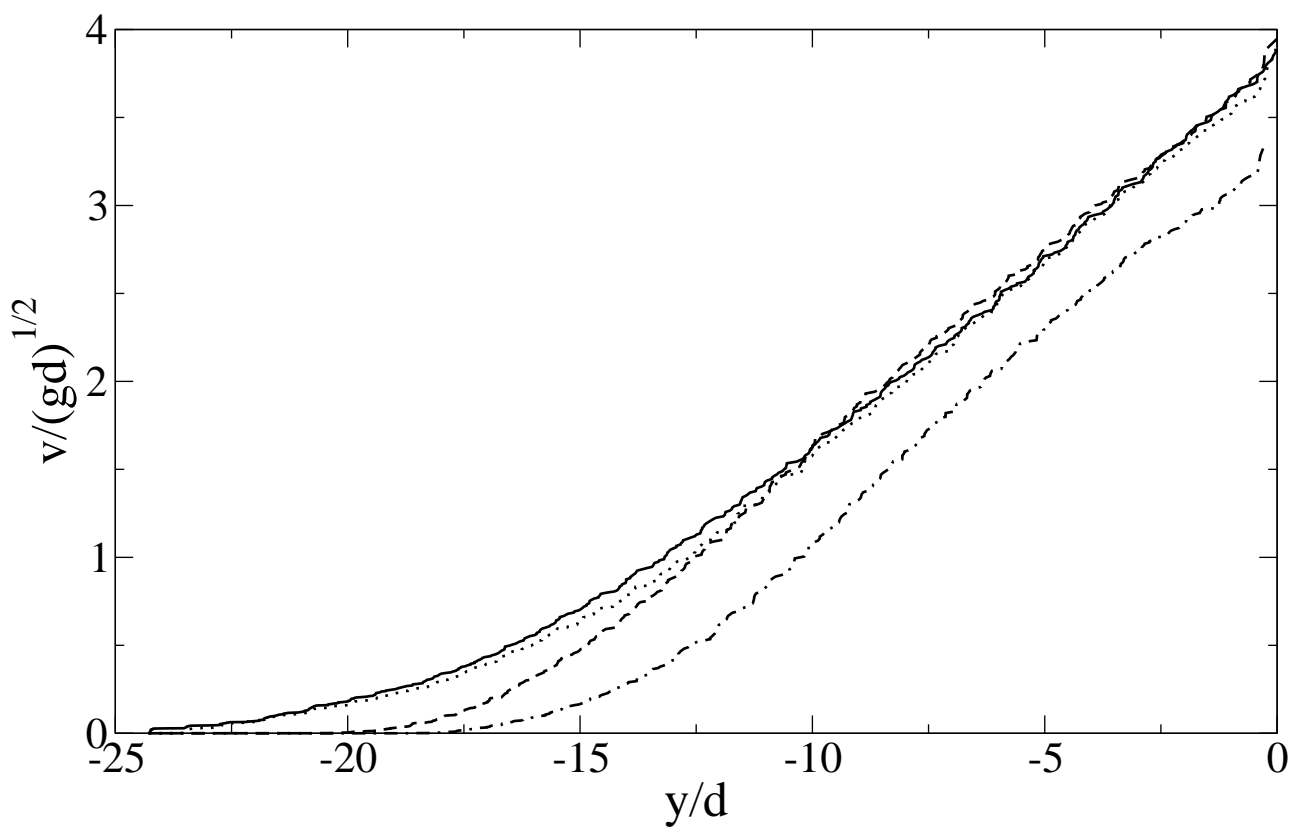

Fig. 7. Velocity profile in the reference frame of the rotating drum for different values of the wake-up threshold: $V_{\text {wakeup }}=0.01 \sqrt{g d}$ (solid line), $V_{\text {wakeup }}=0.05 \sqrt{g d}$ (dotted line), $V_{\text {wakeup }}=0.25 \sqrt{g d}$ (dashed line), $V_{\text {wakeup }}=0.50 \sqrt{g d}$ (dot-dashed line). Distances are measured in a thin slab, perpendicular to the free surface in the center of the system, with the origin placed at the free surface.

When the wake up threshold is $0.50 \sqrt{g d}$ (equivalent to waking a particle up if it is hit by another particle that fell from a height of $d / 8$ ) the velocity profile resembles those found experimentally [19. The system develops a continuous avalanche of a thickness of approximately two thirds of the drum radius and the rest of the grains remain motionless with respect to the drum. Due to our resolution, we cannot affirm that the transition region has an exponential decay.

However, when the wake up threshold is too small $(0.05 \sqrt{g d})$, the particles near the drum wall get overfluidized and there is no frozen region. In fact, because of the regularization, particles near the bottom do not sleep because they have not reached this threshold but collisions are already elastic. Therefore they remain with a finite amount of energy and the bottom region is in an artificial elastic regime. This result shows that the IHS model is inaccurate to describe dense granular regions since the regularization method, introduced to avoid the inelastic collapse, creates artificially overfluidized elastic regions. Jamming and freezing is not possible and solid like regions become softer. Our model corrects this by providing an additional method to dissipate energy -freezing the particles- allowing for a better description of dense flows near a fixed boundary. Further research, however, is needed to determine criteria to fix $V_{\text {wakeup }}$. 


\section{Conclusions}

We have extended the IHS model for granular media to include static particles that have lost all its kinetic energy, allowing to simulate granular flows coexisting with solid-like regions. It allows to reach the static limit, recreating multiple persisting contacts. The model is efficient because collision events are only computed for particles that are moving, which are the only dynamically relevant. A combination of strategies to sleep, wake up, and self-check particles allows to dynamically move particles to frozen and normal states back and forth. The model has been used to study two cases that present difficulties when studied using the standard or regularized IHS model: the homogeneous deposition until all particles are motionless and the continuous avalanche that develops in a rotating drum. Our model correctly describes these cases with results that agree with those found experimentally. Besides, sleeping the particles provides with an additional energy dissipation mechanism, that is important to describe correctly flows near a fixed boundary.

The model uses the parameter $V_{c}$ that has been widely discussed in the literature and three new parameters: $V_{\text {sleep }}, V_{\text {wakeup }}$, and $\alpha_{\text {frozen }}$. The effect of the two threshold velocities $V_{\text {sleep }}$ and $V_{\text {wakeup }}$ have been discussed in the two examples. Nevertheless, an estimation for $\alpha_{\text {frozen }}$ needs a model for the interaction of a grain with a frozen region, which for small velocities is lacking. In absence of more information $\alpha_{\text {frozen }}$ was fixed arbitrary. Further research is needed.

We mention that as in the two setups we considered, frozen particles that cannot wake up can be used to build up complex boundaries with no additional computational cost.

We thank E. Clement and P. Cordero for fruitful discussions and S. Pérez for critical reading the manuscript. This research is supported by Fondecyt Grants No. 1061112, No. 1070958, and Fondap Grant No. 11980002.

\section{References}

1. M. P. Allen and D.J. Tildesley, Computer Simulation of Liquids Clarendon Press, 1987.

2. J.-P. Hansen and I.R. McDonald Theory of Simple Liquids, 2nd ed., Academic Press, London, 1986.

3. S. Torquato, Random Heterogeneous Materials, Springer (2001).

4. J.T. Jenkins and Chao Zhang, Phys. Fluids 14 1228, (2002).

5. C.S. Campbell, Annu. Rev. Fluid Mech. 22, 57 (1990).

6. H.M. Jaeger, S.R. Nagel, and R.P. Behringer, Rev. Mod. Phys. 68, 1259 (1996).

7. A. Kudrolli, Rep. Prog. Phys. 67, 209 (2004).

8. M. Marín, D. Risso, and P. Cordero, J. Comput. Phys. 109, 306 (1993).

9. S. McNamara and W. R. Young, Phys. Rev. E 50, R28 (1994).

10. S. Luding, and S. McNamara, Granular Matter 1, 113, (1998).

11. T. Pöschel and T. Schwager, Computational Granular Dynamics: Models and Algorithms, Springer (Berlin 2005).

12. J.J. Moreau, Non-smooth Mechanics and Applications, CISM Courses and Lectures, vol. 302, Springer, Wien (1988).

13. The inelastic collapse is also found in the IHS model with a restitution coefficient that approaches one for small velocities, like in R. Ramírez, T. Pöschel, N.V. Brilliantov, and T. Schwager, Phys. Rev. E 60, 4465 (1999), although the scaling for the inelastic collapse time is different.

14. W. M. Visscher and M. Bolsterli. Nature, 239, 504 (1972).

15. J. Crassous, D. Beladjine, and A. Valance, Phys. Rev. Lett. 99, 248001 (2007). D. Beladjine, M. Ammi, L. Oger, and A. Valance, Phys. Rev. E 75, 061305 (2007).

16. S. Torquato, T. M. Truskett, and P. G. Debenedetti, Phys. Rev. Lett. 84, 2064 (2000).

17. L. Vanel, D. Howell, D. Clark, R.P. Behringer, E. Clément, Phys. Rev. E 60, R5040 (1999).

18. G.Y. Onoda and E.G. Liniger, Phys. Rev. Lett. 64, 2727 (1990).

19. GDR MiDi, Eur. Phys. J. E 14, 341 (2004). 